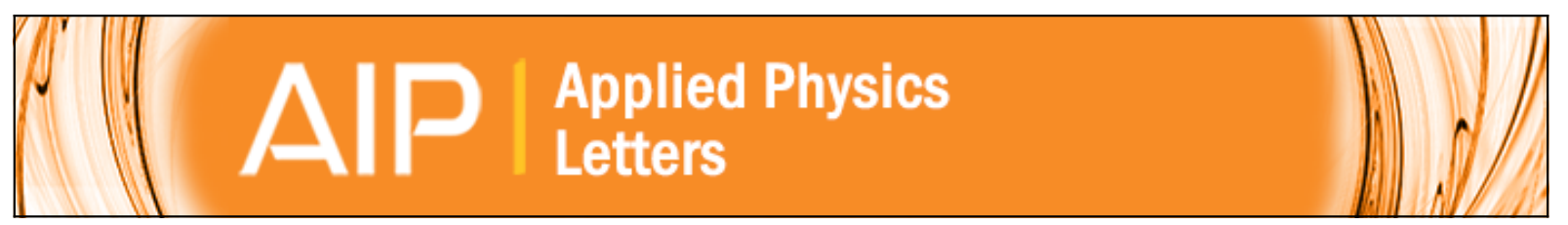

\title{
Post-processing approach for tuning multi-layered metamaterials
}

Liming Liu, Wen-chen Chen, David A. Powell, Willie J. Padilla, Fouad Karouta, Haroldo T. Hattori, Dragomir N.

Neshev, and llya V. Shadrivov

Citation: Applied Physics Letters 105, 151102 (2014); doi: 10.1063/1.4897949

View online: http://dx.doi.org/10.1063/1.4897949

View Table of Contents: http://scitation.aip.org/content/aip/journal/apl/105/15?ver=pdfcov

Published by the AIP Publishing

\section{Articles you may be interested in}

Ultra-broadband terahertz metamaterial absorber

Appl. Phys. Lett. 105, 021102 (2014); 10.1063/1.4890521

Tuning characteristics of mirrorlike T-shape terahertz metamaterial using out-of-plane actuated cantilevers Appl. Phys. Lett. 104, 251914 (2014); 10.1063/1.4885839

Tunable terahertz left-handed metamaterial based on multi-layer graphene-dielectric composite Appl. Phys. Lett. 104, 051902 (2014); 10.1063/1.4863929

Enhanced mass transport in ultrarapidly heated $\mathrm{Ni} / \mathrm{Si}$ thin-film multilayers

J. Appl. Phys. 106, 104909 (2009); 10.1063/1.3254225

Prediction of Ultrasonic Fields into Composite MultiLayered Structures: Homogenization Approach for the Direct Field and Statistical Approach for the Inner Reflections

AIP Conf. Proc. 657, 957 (2003); 10.1063/1.1570237

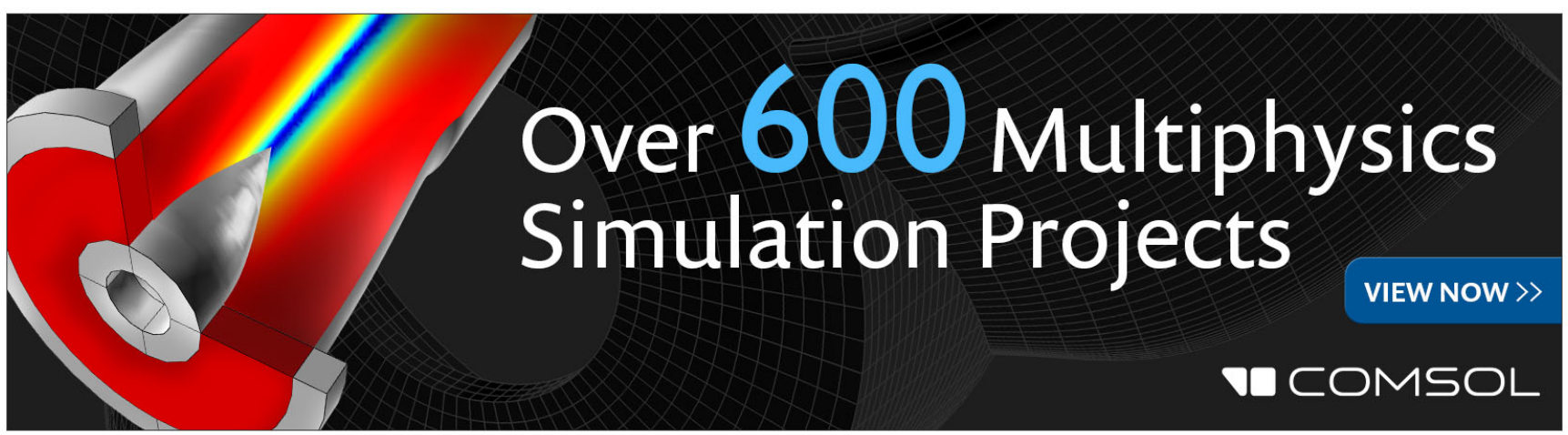




\title{
Post-processing approach for tuning multi-layered metamaterials
}

\author{
Liming Liu, ${ }^{1,2, a)}$ Wen-chen Chen, ${ }^{3}$ David A. Powell, ${ }^{4}$ Willie J. Padilla, ${ }^{3}$ Fouad Karouta, ${ }^{5}$ \\ Haroldo T. Hattori, ${ }^{2}$ Dragomir N. Neshev, ${ }^{4}$ and Ilya V. Shadrivov ${ }^{1}$ \\ ${ }^{1}$ Nonlinear Physics Centre, Research School of Physics and Engineering, Australian National University, \\ Canberra, Australia \\ ${ }^{2}$ School of Engineering and Information Technology, University of New South Wales, ACT 2612, Australia \\ ${ }^{3}$ Department of Physics, Boston College, 140 Commonwealth Ave., Chestnut Hill, Massachusetts 02467, USA \\ ${ }^{4}$ Nonlinear Physics Centre and Centre for Ultrahigh bandwidth Devices for Optical Systems (CUDOS), \\ Research School of Physics and Engineering, Australian National University, Canberra, Australia \\ ${ }^{5}$ Australian National Fabrication Facility, Department of Electronic Materials Engineering, Research School \\ of Physics and Engineering, The Australian National University, Canberra, ACT 0200, Australia
}

(Received 7 August 2014; accepted 19 September 2014; published online 13 October 2014)

We propose a post-processing approach to efficiently tune the resonance frequency in doublelayered terahertz metamaterials separated by a bonding agent. By heating the bonding agent, it is possible to move one metamaterial layer laterally with respect to the other. This changes the coupling between adjacent layers, thereby shifting the resonance frequency. The resonance frequency of the stacked layers continuously shifts as a function of the lateral displacement, reaching a maximum shift of $92 \mathrm{GHz}$ ( $31 \%$ of the center frequency). We discuss the effects of vertical separation on the tunability of the two-layered structure. The post-processing approach is rather general and can be applied to different paired metamaterials in various wavelength ranges, paving the way to efficiently assemble and fine tune metamaterial sensors and filters. (C) 2014 AIP Publishing LLC.

[http://dx.doi.org/10.1063/1.4897949]

Metamaterial research has reached the stage where scientists and engineers are looking for applications of the knowledge generated over the past decades. Devices based on metamaterials need to meet certain performance specifications which are inevitably affected by fabrication tolerances. Metamaterials are often made from subwavelength elements, therefore fabrication processes for shorter wavelengths of the spectrum, including terahertz (THz) and optical frequencies, are often challenging and the precision of the produced elements is not always high. To eliminate the requirement for multiple fabrication runs in order to achieve the required performance of metamaterials, we propose a post-processing technique that allows fine tuning metamaterial properties after they are fabricated. Our proposed technique can also be used to create tunable filters and other devices, since it has proven to have a large tunability range.

Different approaches have been implemented to realize metamaterial resonance tuning, in most cases by changing the metamaterial geometry ${ }^{1,2}$ or the surrounding medium. ${ }^{3}$ Examples of resonance tuning include adjusting the lattice structure manually, ${ }^{1}$ stretching metamaterials made on an elastic substrate, ${ }^{4}$ introducing ferromagnetic elements, ${ }^{5}$ lumped capacitors, ${ }^{6}$ liquid crystals, ${ }^{7}$ or phase change materials. ${ }^{8}$ At terahertz frequencies, metamaterials can be tuned by infiltrating them with liquid crystals ${ }^{9}$ and also by integrating graphene ${ }^{10}$ or semiconductor ${ }^{11}$ layers and subsequently applying a voltage or changing the temperature of the device. Since the dimensions of $\mathrm{THz}$ metamaterials are generally in the range of $30-300 \mu \mathrm{m}$, it is natural to embrace microelectromechanical (MEMS) technologies to produce tunable devices. The benefits of using MEMS include the potential to achieve

\footnotetext{
${ }^{\text {a) }}$ Author to whom correspondence should be addressed. Electronic mail: liming.liu@student.adfa.edu.au
}

dynamic tunability and integration of multiple devices on the same chip. ${ }^{12,13}$ This approach utilizes the established mechanism to achieve tunability by adjusting the near-field interaction between adjacent metamaterial layers. ${ }^{14,15}$

Dynamical tunability provided by MEMS is not always required, but a post-processing change of metamaterial parameters can ensure that it meets the required specifications. In this letter, we present a post-processing approach based on near-field coupling between double-layered metamaterials to achieve the tuning of the resonance frequency. We fabricate two metamaterials separately, and then use a bonding agent (Epikote828) to attach them together. This glue can be melted at temperatures above $90^{\circ} \mathrm{C}$, providing the flexibility of moving the metamaterial layers laterally with respect to each other. Once the desired arrangement is achieved, the relative position between the adjacent layers can be maintained by cooling the structure back to room temperature. This approach can be used to achieve different spacings or lateral displacements in a double-layered metamaterials leading to different electromagnetic response without having to fabricate multiple samples.

The building block of the metamaterial layers is an electric field-coupled LC resonator, which can be considered as a pair of connected split ring resonators, as shown in Fig. 1. As the resonators in neighbouring cells are electrically connected, the structure exhibits substantial transmission only near its resonances. The metamaterial layers are fabricated using conventional optical lithography followed by a lift-off process. The thickness of the gold is $150 \mathrm{~nm}$, and the resonators are arranged in a square lattice with the lattice constant of $135 \mu \mathrm{m}$. The overall structure occupies an area of $5 \mathrm{~mm}$ by $5 \mathrm{~mm}$. After two metamaterial samples are fabricated, a small amount of solidified glue is put on top of each sample and then heated until it reaches the melting point. The two 
metamaterial layers are face-to-face bonded and aligned with each other under a microscope. The lateral displacement can be varied by re-heating the sample and moving the top layer with respect to the bottom one. The spacing between the two layers can be controlled by moving the top layer back and forth to redistribute the glue. Tunability is achieved by controlling the coupling between the two adjacent metamaterial structures: The frequency shift is controlled by moving the structures laterally, while the maximum frequency shift is controlled by the thickness of the glue. The dimensions of the resonators shown in Fig. 1(a) are $W_{1}=8 \mu \mathrm{m}, W_{2}=15 \mu \mathrm{m}$, $W_{3}=65 \mu \mathrm{m}, L_{1}=27 \mu \mathrm{m}, L_{2}=19 \mu \mathrm{m}$, and $X=Y=135 \mu \mathrm{m}$. Figs. 1(c) and 1(d) show the schematics of the double-layered structure. The lateral displacements can be changed from $S=0 \mu \mathrm{m}$ to half unit cell $S=67.5 \mu \mathrm{m}$.

Numerical simulations are performed by using commercial electromagnetic solver CST Microwave Studio. In our simulations, we have used normally incident waves polarized across the gap of the resonator. The refractive index for Epikote828 is 1.6 (Ref. 16) in the simulation. It is assumed that the structures are periodic in the $x-y$ plane, and the computational area is terminated by perfectly matching boundary layers in the $\mathrm{z}$ direction. The spacing between the metamaterial layers is determined by measuring the optical transmission at the edges of the fabricated samples outside the metamaterial region. The frequency spacing between Fabry-Perot transmission fringes depend on the distance between the plates. The averaged spacing for six positions measured around the metamaterial region is $10.4 \mu \mathrm{m}$ with variations below $2.5 \mu \mathrm{m}$.

Transmission through the double-layered metamaterials is measured by using a Terahertz time-domain spectroscopy (THz-TDS) system from EKSPLA. The polarization of the incident field is aligned across the gaps of the resonators. The system is excited by a femtosecond pulse, and the transmission response is sampled in the time domain. A Fast Fourier Transform (FFT) is used to obtain the amplitude and phase of the transmitted wave and further signal processing in the frequency domain allows the calculation of the experimental transmission spectrum. Fabry-Perot interference fringes in the transmission spectrum are reduced by employing time-gating in the time-domain signal.

The measured resonance frequency for the single layer structure is $0.375 \mathrm{THz}$ compared with the theoretical value of $0.386 \mathrm{THz}$ obtained in simulations, as shown in Fig. 1(b). This difference is due to variations of the designed dimensions after fabrication of the devices. In any case, there is a good agreement between the theoretical and experimental results. The bandwidth of the resonance is about $0.17 \mathrm{THz}$. The experimental and simulation results for the doublelayered structure with different lateral displacements are shown in Fig. 2 for a separation of $10.4 \mu \mathrm{m}$. The alignment is also shown for different lateral displacements. The alignment achieved by manual adjustment of alignment marks under the microscope is reasonably good, demonstrating the feasibility of the proposed post-processing approach.

As shown in the second row of Fig. 2(a), two resonances $(0.318 \mathrm{THz}$ and $0.468 \mathrm{THz})$ are observed for coupled resonators in simulation when $S=0 \mu \mathrm{m}$. The electric resonators are excited by the $\mathrm{THz}$ field, and the generated charges and currents are strongly coupled between adjacent resonators in two parallel layers, and this coupling generates symmetric and antisymmetric modes. ${ }^{15,17,18}$ The current distributions are shown in Fig. 3 for the top and bottom layer at the two resonances. The currents (and, thus, charges) at the lower resonance $\omega_{-}(0.318 \mathrm{THz})$ are in phase between the top and bottom metamaterial layers and are out of phase at the higher resonance $\omega_{+}(0.468 \mathrm{THz})$. We interpret the frequency splitting of these coupled modes in terms of competing electric and magnetic interaction terms, ${ }^{15}$ and note that for this configuration of charges and currents, both interaction terms are positive. Therefore, the numerically and experimentally observed negative frequency shift indicates that the magnetic (a)

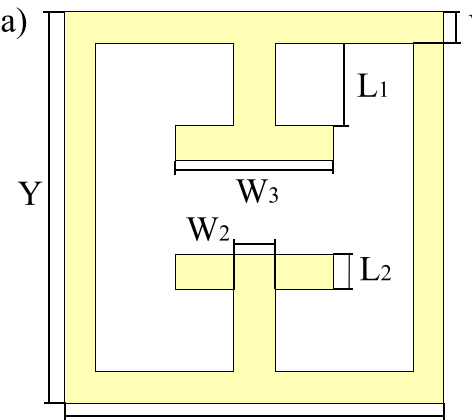

(c)

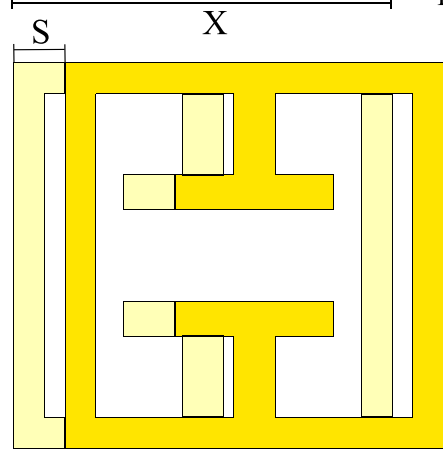

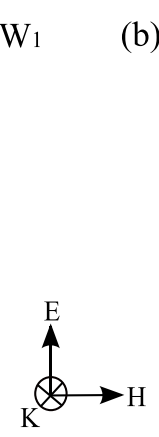

(d)
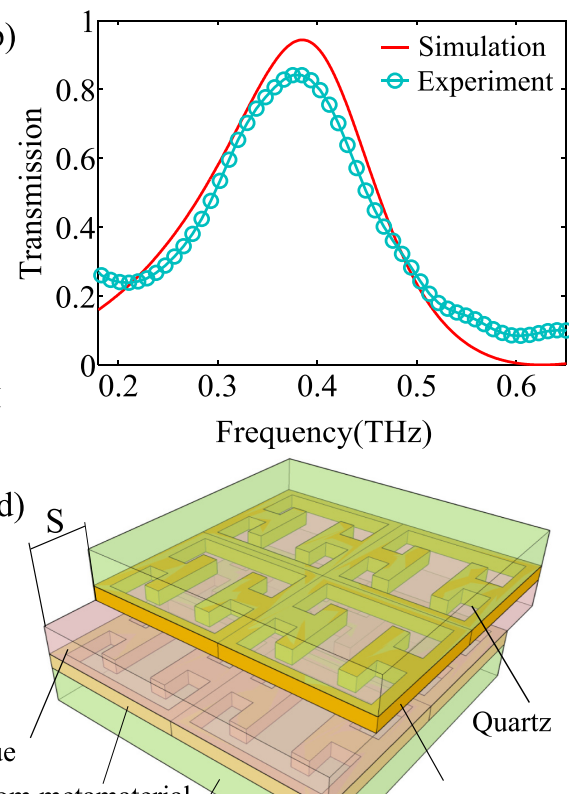

Bottom metamaterial layer

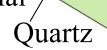

FIG. 1. (a) Geometry of metamaterial structure including the wave polarizations. (b) Simulated and experimentally measured transmission for a single layer. (c) Schematics of the double-layered structure (top view). (d) Schematics of the double-layered structure with substrate and superstrate (front view). 


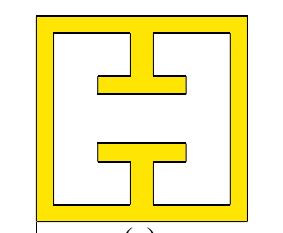

(a)

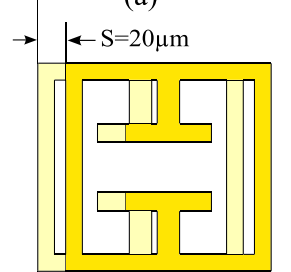

(b)

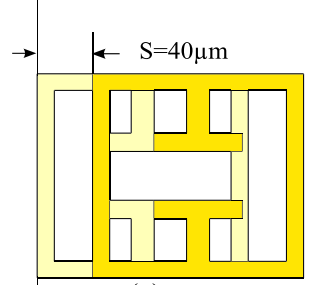

(c)

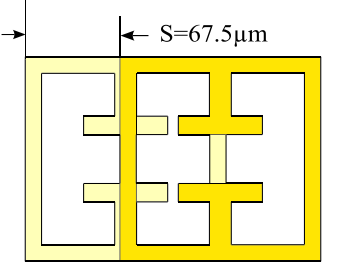

(d)
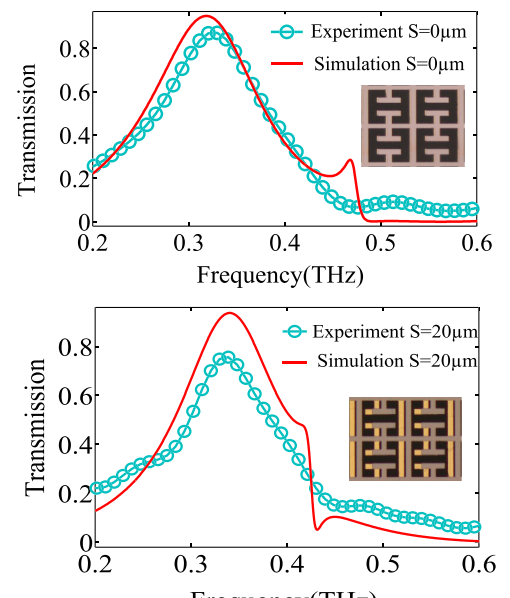

Frequency(THz)
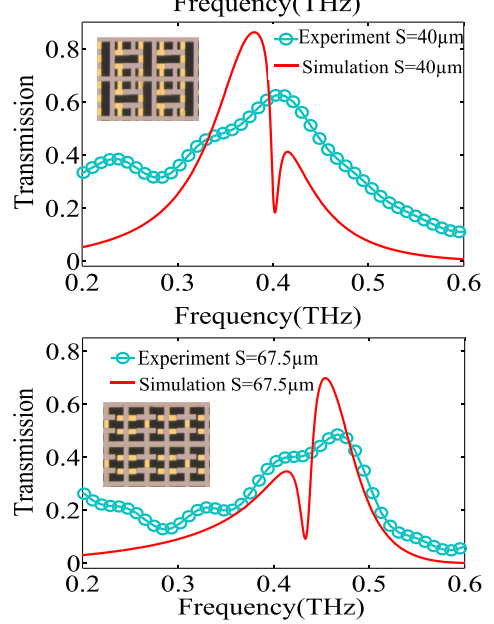

FIG. 2. Schematics, measurement versus simulation results, and alignment taken under microscope (inset) of the double-layered structure when (a) $S=0 \mu \mathrm{m}$, (b) $S=20 \mu \mathrm{m}$, (c) $S=40 \mu \mathrm{m}$, and (d) $S=67.5 \mu \mathrm{m}$.

interaction is stronger. We limit our analysis to the symmetric mode, as it has the much stronger response which is more clearly observable in the experimental spectrum.

When $S$ is varied from $0 \mu \mathrm{m}$ to $67.5 \mu \mathrm{m}, \omega_{-}$shifts from $0.318 \mathrm{THz}$ to $0.416 \mathrm{THz}$ which is a frequency shift of nearly $100 \mathrm{GHz}$ (31\% of the resonance). For experimental measurements of the double-layered structure shown in circle-line in Fig. 2, it is hard to observe the antisymmetric resonance $\omega_{+}$ for small lateral displacements. This is due to $\omega_{+}$having small magnitude, and insufficient frequency resolution in the experiment, which is impaired by Fabry-Perot resonances in metamaterial substrates. Figure 4 shows resonance shifts

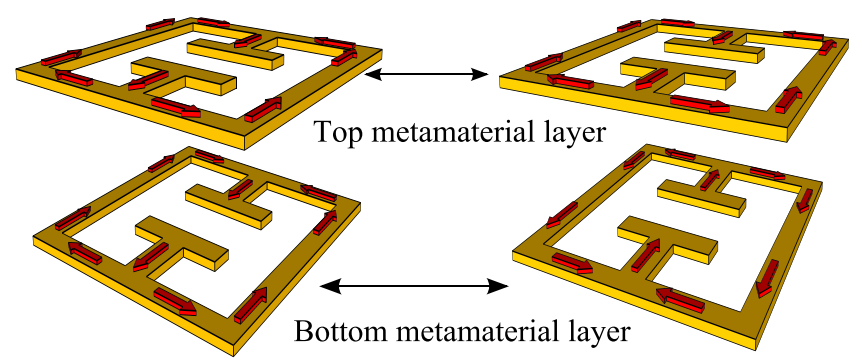

(a)

(b)

FIG. 3. Current distributions of top and bottom metamaterial layer (a) at lower resonance $\omega_{-}=0.318 \mathrm{THz}$ and $(\mathrm{b})$ at higher resonance $\omega_{+}=0.468$ $\mathrm{THz}$. Arrow indicates the current direction.

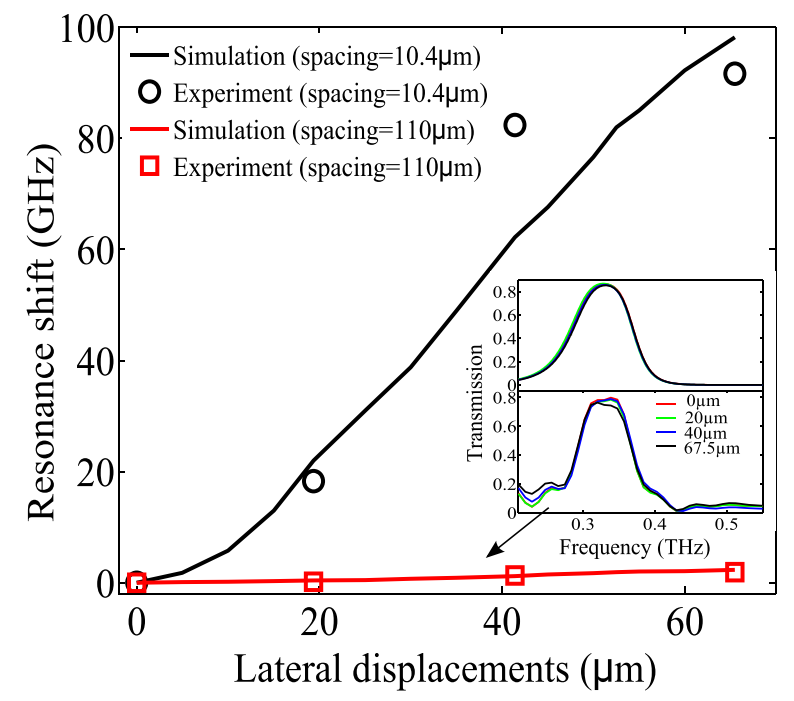

FIG. 4. Resonance tunability with respect to lateral displacements in small spacing $(10.4 \mu \mathrm{m})$ and $\mathrm{THz}$ transmission (inset) measurement of larger spacing $(110 \mu \mathrm{m})$ under different lateral displacements.

with respect to lateral displacements when spacing between two layers is $10.4 \mu \mathrm{m}$. There is a good agreement between simulation and experiment, and an experimental resonance shift of $92 \mathrm{GHz}$ is comparable to $100 \mathrm{GHz}$ in simulation.

The effect of the lateral shift is to decrease both coupling terms at different rates, thus changing the splitting between symmetric and anti-symmetric modes. The magnetic coupling term is expected to undergo the greatest shift, which can be understood by comparing Figs. 2(a) and 2(d). By noting the symmetric current distribution in Fig. 3(a), it is clear that the nearest current loops are now out of phase, giving a strong negative contribution to the magnetic interaction. In addition, the height of the resonant peak is also reduced, which indicates a smaller oscillator strength.

We also simulate the resonance shift for larger spacing of $110 \mu \mathrm{m}$ shown in inset of Fig. 4. For the large spacing, the vertical coupling between two layers is significantly reduced. In the limits of larger spacing, the resonance of stacked layers will closely resemble isolated layers of metamaterials due to the absence of strong interactions between them. ${ }^{17}$ Hence, in this case the lateral displacement will induce negligible resonance tuning, which is clearly shown in Fig. 4. It is noted that the double-layered structure resonates at a lower frequency than the single layer structure due to the presence of the glue layer in between.

In conclusion, we have demonstrated a post-processing approach for double-layered tunable $\mathrm{THz}$ metamaterials. By re-heating the adhesive layer between them, the lateral displacement between the top and bottom layer could be adjusted manually, thus changing the coupling strength. Resonance tunability of $92 \mathrm{GHz}$ was achieved experimentally for a spacing of $10.4 \mu \mathrm{m}$. Coupling between the adjacent metamaterial layers is substantially reduced when the spacing is increased to $110 \mu \mathrm{m}$, and thus negligible resonance tunability was observed. This post-processing approach may find applications in building tunable $\mathrm{THz}$ devices without involving complex fabrication processes and can lead to a large tunability in a pair of layers. 
The authors would like to acknowledge the financial support provided by the Australian Research Council and the Asian Office of Aerospace Research and Development-U.S. Air Force. We also acknowledge the technical support from the Australian National Fabrication Facility, ACT Node.

${ }^{1}$ M. Lapine, D. A. Powell, M. V. Gorkunov, I. V. Shadrivov, R. Marqués, and Y. S. Kivshar, Appl. Phys. Lett. 95, 084105 (2009).

${ }^{2}$ W. Withayachumnankul, C. Fumeaux, and D. Abbott, J. Phys. D: Appl. Phys. 45, 485101 (2012).

${ }^{3}$ D. Shrekenhamer, W.-C. Chen, and W. J. Padilla, Phys. Rev. Lett. 110, 177403 (2013).

${ }^{4}$ J. Li, C. M. Shah, W. Withayachumnankul, B. S.-Y. Ung, A. Mitchell, S. Sriram, M. Bhaskaran, S. Chang, and D. Abbott, Appl. Phys. Lett. 102, 121101 (2013).

${ }^{5}$ L. Kang, Q. Zhao, H. Zhao, and J. Zhou, Opt. Express 16, 8825 (2008).

${ }^{6}$ O. Reynet and O. Acher, Appl. Phys. Lett. 84, 1198 (2004).

${ }^{7}$ A. Minovich, J. Farnell, D. N. Neshev, I. McKerracher, F. Karouta, J. Tian, D. A. Powell, I. V. Shadrivov, H. Hoe Tan, C. Jagadish, and Y. S. Kivshar, Appl. Phys. Lett. 100, 121113 (2012).
${ }^{8}$ M. J. Dicken, K. Aydin, I. M. Pryce, L. A. Sweatlock, E. M. Boyd, S. Walavalkar, J. Ma, and H. A. Atwater, Opt. Express 17, 18330 (2009).

${ }^{9}$ L. Liu, I. V. Shadrivov, D. A. Powell, R. Raihan, H. T. Hattori, M. Decker, E. Mironov, and D. N. Neshev, IEEE Trans. Terahertz Sci. Technol. 3, 827 (2013).

${ }^{10}$ L. Ju, B. Geng, J. Horng, C. Girit, M. Martin, Z. Hao, H. A. Bechtel, X. Liang, A. Zettl, Y. R. Shen, and F. Wang, Nat. Nanotechnol. 6, 630 (2011).

${ }^{11}$ H.-T. Chen, J. F. O'Hara, A. K. Azad, A. J. Taylor, R. D. Averitt, D. B. Shrekenhamer, and W. J. Padilla, Nat. Photonics 2, 295 (2008).

${ }^{12}$ Y. H. Fu, A. Q. Liu, W. M. Zhu, X. M. Zhang, D. P. Tsai, J. B. Zhang, T. Mei, J. F. Tao, H. C. Guo, X. H. Zhang, J. H. Teng, N. I. Zheludev, G. Q. Lo, and D. L. Kwong, Adv. Funct. Mater. 21, 3589 (2011).

${ }^{13}$ N. I. Zheludev and Y. S. Kivshar, Nat. Mater. 11, 917 (2012).

${ }^{14}$ E. Ekmekci, A. Strikwerda, K. Fan, G. Keiser, X. Zhang, G. TurhanSayan, and R. D. Averitt, Phys. Rev. B 83, 193103 (2011).

${ }^{15}$ D. A. Powell, M. Lapine, M. V. Gorkunov, I. V. Shadrivov, and Y. S. Kivshar, Phys. Rev. B 82, 155128 (2010).

${ }^{16}$ H. Tsuda and K. Urabe, Sensors 9, 4559 (2009).

${ }^{17}$ N. Liu, H. Guo, L. Fu, S. Kaiser, H. Schweizer, and H. Giessen, Nat. Mater. 7, 31 (2008).

${ }^{18}$ M. T. Reiten, D. Roy Chowdhury, J. Zhou, A. J. Taylor, J. F. O'Hara, and A. K. Azad, Appl. Phys. Lett. 98, 131105 (2011). 